\title{
Relación entre el estilo de liderazgo del director escolar y la motivación de los maestros y maestras de la generación millennials
}

\author{
MELISSA HERNÁNDEZ COLÓN \\ A plus Educational and Consulting Solutions - Puerto Rico \\ Recibido el 30-03-20; evaluado el 08-01-21; \\ aceptado el 28-01-21
}

\section{RESUMEN}

El propósito de esta investigación cuantitativa correlacional fue determinar cómo se relaciona el estilo de liderazgo del director escolar con la motivación laboral de los empleados de la generación millennials. El marco teórico que fundamentó el problema de investigación fue la teoría existencialista humanista de Ortega. La muestra la conformaron 176 participantes de la región de Ponce del Departamento de Educación de Puerto Rico. Para recopilar los datos, se utilizó el instrumento de motivación extrínseca e intrínseca, y el Análisis del comportamiento del líder II (LBAII, por sus siglas en inglés). De los datos, se estableció un perfil de los participantes del estudio. El resultado principal arrojó que existe una relación significativa entre el estilo de liderazgo del director con la motivación de los maestros y maestras de la generación millennials.

Palabras claves: estilo de liderazgo del director escolar, motivación laboral, generación millennials

\footnotetext{
* Con licenciatura en Pedagogía, maestría en Administración y Supervisión Escolar y doctorado en Gerencia y Liderazgo Educativo por la Pontificia Universidad Católica de Puerto Rico, Recinto de Ponce. Ha ejercido como maestra y directora escolar en el Departamento de Educación de Puerto Rico. Actualmente se desempeña como ejecutiva de cuentas y consultora educativa de la compañía A plus Educational and Consulting Solutions. Correo electrónico: melissahernandez2@pucpr.edu. https://orcid.org/0000-0001-6782-2787
} 


\section{The relationship between the leadership style of the director and the motivation of millennial generation teachers}

\section{Abstract}

The purpose of this quantitative correlational research was to determine how the leadership style of the school principal relates to the work motivation of Millennials Generation employees. The theoretical framework that founded the research problem was the existentialist humanist theory of Ortega. The sample was made up of 176 participants from the Ponce region of the Puerto Rico Department of Education. To collect the data, the extrinsic and intrinsic motivation instrument, and the Leader Behavior Analysis II (LBAII) were used. From the data, a profile of the study participants was established. The main result showed that there is a significant relationship between the leadership style of the director and the motivation of the Millennials generation teachers.

Keywords: principal leadership style, work motivation, millennials generation

\section{Relaçáo entre o estilo de liderança do diretor da escola e a motivaçáo dos funcionários da geraçáo milenar}

\section{Resumo}

O objetivo desta pesquisa correlacional quantitativa foi determinar como o estilo de liderança do diretor da escola se relaciona com a motivação para o trabalho dos funcionários da Geração Millennials. O referencial teórico que fundou o problema de pesquisa foi a teoria humanista existencialista de Ortega y Gasset. A amostra foi composta por 176 participantes da regiáo de Ponce, no Departamento de Educação de Porto Rico. Para a coleta dos dados, foram utilizados o instrumento de motivação extrínseca e intrínseca e o Análise do comportamento líder II (LBAII). A partir dos dados, foi estabelecido um perfil dos participantes do estudo. O principal resultado mostrou que existe uma relaçáo significativa entre o estilo de liderança do diretor e a motivação dos funcionários da geração millennials.

Palavras-chave: estilo de liderança do diretor da escola, motivação para o trabalho, geração do milênio

\section{INTRODUCCIÓN}

Es la primera vez en la historia en las organizaciones que se han desarrollado cuatro generaciones en la fuerza laboral (Lowe, Levitt y Wilson, 2008). Estas son: la generación tradicional (nacidos antes de 1945), los baby boomers (1945-1964), la generación X (1965-1980) y, finalmente, la generación millennials, que son los nacidos luego del 1980. Esta situación implica que el líder o gerente de la institución debe poseer las estrategias para trabajar con 
todas las generaciones, aunque esto represente diferentes características, visiones del trabajo y la espera de recompensas por el trabajo realizado.

La sociología se ha dedicado al estudio de las generaciones y, a partir del siglo XX, han identificado cuatro, que son la generación tradicional, los baby boomers, la generación X y la generación millennials. Los investigadores han variado en los intervalos de años de nacimiento, por lo que no hay unanimidad, por tanto, en las diversas investigaciones que se han realizado y que aquí se presentarán se puede apreciar la incongruencia en las fechas (Naim y Lenka, 2018). Es por esta razón que, además de las fechas de nacimientos, se aprecia también cómo se utilizan hechos históricos o trascendentales para ubicar a las personas dentro de una generación.

El problema que presenta esta investigación es acerca de las diferencias generacionales que comparten el ambiente de trabajo y el estilo de liderazgo que posee el director escolar y su relación con la motivación de los empleados millennials. Esto llevó a delinear como propósito determinar cómo se relaciona el estilo de liderazgo del director escolar con la motivación laboral de los empleados de la generación millennials En una encuesta realizada por Virtuali, explicado por Agüero (2015), casi la mitad de los 412 millennials encuestados (47\%) dijeron que se ven motivados a ser líderes porque quieren empoderar a otros, mientras que, solo el $10 \%$ se preocupó por el legado y el $5 \%$ manifestó que asumiría un puesto de liderazgo por el dinero.

Los miembros de la generación millennials especularon que las habilidades blandas (soft skills) los ubicarán en el camino rápido hacia los puestos de liderazgo. Los participantes de la encuesta indicaron que las destrezas y competencias más importantes son la comunicación (58\%) y las relaciones interpersonales (55\%) y sienten que ya dominan en ambas áreas. También, poseen menos confianza en su conocimiento de la industria (43\%) y su experiencia técnica (41\%) (Agüero, 2015). Más del 50\% prefirió no tener empleo a permanecer en un trabajo que odien. Aunque parezca que son tan egocéntricos, en realidad ocho de cada diez participantes de la encuesta indicaron que necesitan retroalimentación de sus supervisores, ya que piensan que es la única forma de saber cómo están haciendo sus labores, diferenciándose de las generaciones pasadas, que prefieren lograr sus objetivos de manera solitaria.

$\mathrm{El}$ interés para realizar esta investigación radicó en el conflicto generacional que se presenta en las escuelas, cuando el director escolar encuentra obstáculos en su rol de ser facilitador de los procesos y que, por tal razón, no cuenta con las estrategias necesarias para motivar a los empleados de la generación millennials.

La generación millennials demanda diferentes necesidades de los profesionales de otras edades (Broido, 2004). De acuerdo con Stanford (2017), 
"cada vez más, los millennials son el centro de atención para las empresas y, en solo 10 ańos, esta generación representará el 75\% de la fuerza laboral de Estados Unidos» (p.1). Los millennials requieren de estrategias diferentes a las utilizadas hasta ahora con otras generaciones. La motivación de los trabajadores en el mundo laboral adquiere importancia a partir del siglo XX. De ahí en adelante, se empiezan a integrar disciplinas para trabajar el comportamiento en las organizaciones (Ramírez, Abreu y Badii, 2008).

El éxito de toda organización depende de la unidad de sus maestros y maestras para prosperar a través de su esfuerzo, compromiso, su práctica y persistencia; por lo tanto, la motivación es un tema importante, ya que la competencia del líder incluye la capacidad de motivar a los empleados (Lussier y Achua, 2016). La calidad de la relación de un directivo con un empleado es el elemento más poderoso de motivación para este último. Ello crea una actitud profesional, positiva y respetuosa, y los empleados son más propensos a adoptar un enfoque similar con sus compañeros y disfrutar del trabajo. Está claro que los estilos de gestión y liderazgo que adopta una empresa tendrán un efecto determinante en el nivel de motivación, la moral y la satisfacción laboral de los empleados (Naile y Selecho, 2014).

Es crucial que los maestros conozcan el poder de la motivación, reconozcan su estilo de aprendizaje en el trabajo y que encuentren formas significativas de involucrarse en el liderazgo escolar. Los maestros y maestras de la generación millennials tienen un enorme potencial para hacer una diferencia en la vida de los estudiantes (Williamson y Meyer-Looze, 2010).

Esta investigación contribuye con datos e información para sentar las bases en las organizaciones que les facilite conocer lo particular de la generación millennials y laborar en la concienciación y la capacitación de los que forman parte de dicha institución educativa. Además, esta investigación ayuda a determinar lo que mejor motiva a los empleados en consideración con las culturas imperantes en la organización. Por último, los resultados de esta investigación serán significativos para las instituciones educativas y para el Departamento de Educación de Puerto Rico, puesto que les permitirá conocer si la motivación laboral es la adecuada para el logro de sus objetivos.

\section{Marco teórico}

El marco teórico que sirvió como base del problema de investigación es la teoría existencialista humanista de Ortega (1983). Esta, como un estudio interdisciplinario, se refiere a los campos de la historia, la sociología, la psicología, la economía, la antropología, la biología y muchas otras disciplinas. 
Para identificar los estilos de liderazgo, se utilizaron los enfoques teóricos del liderazgo transformativo-transaccional de Bass y Avolio (1994), el estilo de liderazgo permisivo (Laissez-faire) de Lewin, Lippit y White (1939) y la teoría de liderazgo situacional de Hersey y Blanchard (1977). Igualmente, se utilizó la Teoría de liderazgo colaborativo de Archer y Cameron (2013).

La teoría existencialista humanista de las generaciones, según Ortega (1983), revela que toda persona lleva consigo un afán de ser y cada generación va actualizándose a partir de la anterior. Ortega explica que el ser humano vive instalado en un mundo que lo posiciona para responder a las circunstancias, los problemas y los peligros y que estos los resolverá según sea la seguridad, las inquietudes y las creencias que existan en cada tiempo; todo esto porque el ser humano nace ya instalado dentro de una estructura con la que tiene que convivir, le guste o no. Ortega añade que la estructura de los humanos no es estática; por lo tanto, va modificándose e incorporando nuevos matices para que la historia siga su curso. Esto trae consigo que cambie la historia y se modifique el mundo.

Ortega (1983) indica que el ser humano es el mismo o casi el mismo, lo que se modifican son las circunstancias y las condiciones que hacen que las comunidades se transformen. Asimismo, expresa que cada vida está inmersa en una misma circunstancia de una vida colectiva. Añade que el ser humano, hasta los veinticinco años, solo aprende y recibe noticias de su entorno a través de los maestros, los libros y las conversaciones, conociendo, a través de estos, el mundo vigente. Luego de esta edad, el ser humano se lanza a vivir por su propia cuenta, tomando en cuenta sus dudas, sus problemas y los retos que le toca enfrentar. Asimismo, Ortega (1983) menciona que los seres humanos, aunque son todos contemporáneos dentro de una misma generación, contribuyen a formar al mundo de una estructura diferente.

Para identificar los estilos de liderazgo, se utilizaron los enfoques teóricos del liderazgo transformativo-transaccional de Bass y Avolio (1994), el estilo de liderazgo permisivo (laissez-faire) de Lewin, Lippit y White (1939) y la teoría de liderazgo situacional de Hersey y Blanchard (1977), a la que originalmente se le llama Teoría del ciclo de vida. Esta última se utilizó también como referencia en esta investigación, ya que expone que los gerentes deben manipular diversos estilos de liderazgo de acuerdo con la situación que se presente. También se usó como referencia la teoría de liderazgo colaborativo de Archer y Cameron (2008), quienes la definen como el liderazgo que se aplica considerando los límites y barreras funcionales en las organizaciones. Este es precisamente uno de los puntos que se pretende investigar: si los directores escolares tienen un estilo de liderazgo que pueda cubrir las necesidades 
de la generación millennials. Para presentar esta generación, se utilizó la teoría generacional de Strauss y Howe (1991).

Bass (1985) describe el liderazgo transformativo-transaccional a partir de los efectos que produce el líder sobre sus seguidores. En virtud de estas propiedades, al liderazgo transformativo-transaccional se le suele considerar más amplio y efectivo que el de tipo transaccional (Lupano y Castro, 2005). Muchos estudios empíricos han demostrado que el liderazgo carismático es el componente más influyente entre las dimensiones del liderazgo transformacional (Avolio, Bass y Jung, 1996; Bass y Avolio, 1994). El componente del carisma, generalmente, tiene la correlación más fuerte que cualquier otra dimensión con calificaciones subordinadas de la efectividad del liderazgo y su propia satisfacción (Avolio y Yammarino, 1990). La definición de liderazgo permisivo, acorde con Badford y Lippitt (2005), es aquel en que el líder muestra poca preocupación, tanto por el grupo, como por la tarea. Bass (citado por Vega y Zavala, 2004), lo describe como el estilo menos satisfactorio y efectivo.

Lewin, Lippitt y White (1939) realizaron estudios experimentales en los que se establecieron diferencias entre el estilo de liderazgo autoritario versus el democrático. El autoritario dicta lo que se tiene que hacer y no se preocupa por el desarrollo del grupo, mientras que el democrático (permisivo) toma en cuenta al grupo y sus necesidades. Manifiestan, como resultado de uno de sus estudios experimentales, que el estilo de vida y pensamiento motivado por el líder domina las relaciones entre el grupo y con el líder.

La teoría del liderazgo situacional de Hersey y Blanchard (1977), desafió el saber convencional de que había un estilo de liderazgo que fuera mejor que otro (Torres, 2010). Los autores de esta teoría asumieron la postura de que el estilo de liderazgo más efectivo depende de la situación (Torres, 2010). El método del liderazgo situacional de Hersey y Blanchard (1977) sostiene que los gerentes deben utilizar diversos estilos de liderazgo de acuerdo con la situación existente (Del Valle, 2010). Sobre el liderazgo colaborativo de Archer y Cameron (2013), expusieron que es un liderazgo que promueve la innovación en la organización, pero, también, los saberes cívicos a través de la formación de equipos con los actores gubernamentales y no gubernamentales. Por otra parte, Rubin (2009) definió el liderazgo colaborativo como una «relación útil en el que todas las partes eligen estratégicamente cooperar para lograr un resultado compartido» (p.115.). La siguiente figura presenta las teorías que se utilizaron para realizar esta investigación. 
Figura 1. Marco teórico de la investigación

Estilos de liderazgo
- Transformativo (Bass y
Avolio, 1994)
- Colaborativo (Archer y
Cameron, 2013)
- Permisivo (Lewin,
Lippit y White, 1939)
- Situacional (Hersey y
Blanchard, 1977)

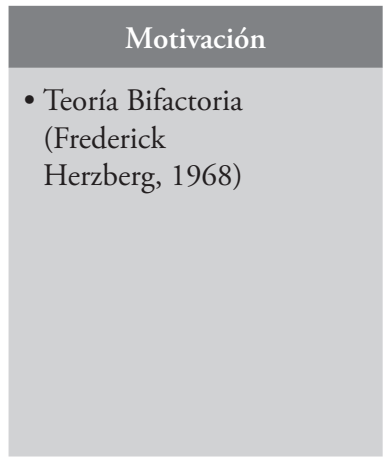

Generación Millennials

- Strauss y Howe (1991)

Fuente: Elaboración propia a partir de Bass y Avolio, (1994), Archer y Cameron, (2013), Lewin, Lippit y White, (1939), Hersey y Blanchard, (1977), Herzberg, (1968) y Strauss y Howe (1991).

\section{Metodología}

\subsection{Diseńo del estudio}

Para el desarrollo de esta investigación se utilizó un enfoque cuantitativo con un diseño correlacional. Para Creswell y Creswell (2017), en los diseños correlacionales se analiza la relación entre variables para entender la incidencia entre las mismas. Este tipo de diseńo fue necesario para analizar la relación entre el estilo de liderazgo del director escolar y la motivación de los maestros de la generación millennials en un contexto investigativo por primera vez.

\subsection{Población y muestra}

Como parte de la población se utilizaron directores, maestros y maestras de todos los niveles de las escuelas públicas de la Región Educativa de Ponce. La selección del tamaño de la muestra se determinó al tomar en cuenta la cantidad de maestros, maestras y directores de la región educativa de Ponce del Departamento de Educación de Puerto Rico. La muestra se escogió de manera no probabilística y por conveniencia. Según Hernández y Mendoza (2018), las muestras no probabilísticas suponen un procedimiento de selección informal y se utilizan en muchas investigaciones. La Tabla 2 ilustra la población de directores, maestros y maestras de todos los niveles en las escuelas públicas de la Región Educativa de Ponce, Puerto Rico, que fueron parte de la investigación. 
Tabla 1. Población de directores, maestros y maestras de todos los niveles en las escuelas públicas de la Región Educativa de Ponce, Puerto Rico

\begin{tabular}{lccc}
\hline \multicolumn{2}{c}{$\begin{array}{c}\text { Cantidad de directores en la región } \\
\text { de Ponce }\end{array}$} & $\begin{array}{c}\text { Número de maestros } \\
\text { y maestras }\end{array}$ & Total \\
\hline Ponce 171 & 1,350 & 1,521 \\
\hline
\end{tabular}

Fuente: Departamento de Educación de Puerto Rico, Oficina de Planificación (2019).

Para establecer el tamaño de la muestra, se utilizó la fórmula otorgada por Krejcie y Morgan (1970), bajo la aplicación de survey monkey. A base de esta fórmula, se seleccionaron 176 participantes entre directores, maestros y maestras de todos los niveles de la región de Ponce del Departamento de Educación de Puerto Rico, de los cuales 54 fueron directores, y 122 fueron maestros y maestras. Esta muestra representativa tuvo un nivel de confianza de un 90\% y un margen de error de 5\%. Los maestros y maestras que fueron parte de la muestra eran nacidos luego del 1980 (millenials). En cuanto a los 54 directores, hubo participación de los Baby Boomers $(f=4,7 \%)$, la Generación X $(f=36$, $67 \%)$ y de la generación millennials $(f=14,26 \%)$.

\subsection{Instrumento para recopilar la información}

Para la construcción del instrumento Relación entre el estilo de liderazgo del director y la motivación de los maestros y maestras de la generación millennials, se tomó como base el del doctor Rubén Rivera Rodríguez, publicado en 2011, con su instrumento de motivación extrínseca e intrínseca aplicado a empleados públicos. Asimismo, a los autores Blanchard, Hambleton, Zigarmi y Drea, con su instrumento de Análisis del comportamiento del líder II (LBAII, por sus siglas en inglés) publicado en 1999.

El instrumento Relación entre el estilo de liderazgo del director y la motivación de los maestros y maestras de la generación millennials constó de tres partes. La parte I recogió datos sociodemográficos y/o personales de la muestra seleccionada. Esta parte tuvo premisas para que el participante seleccionara la respuesta que describiera los siguientes datos sociosdemográficos y/o personales: género, edad, nivel académico alcanzado, ańos de experiencia, entre otros.

La parte II, proporcionó veinticuatro premisas que ayudaron a describir la motivación extrínseca e intrínseca que tiene el participante. Esta parte se midió mediante una escala Likert, donde el participante contestaba si estaba totalmente en desacuerdo, muy en desacuerdo, de acuerdo, muy de acuerdo o totalmente de acuerdo en la motivación. 
La parte III constó de veinte premisas que representaron situaciones laborales típicas que involucran al líder y a uno o más de sus colaboradores directos, los cuales presentan como alternativas cuatro, tres y dos posibles respuestas. Estas alternativas buscaban describir la autopercepción que tenían los supervisores, en este caso, de sus cualidades de liderazgo con tres propósitos básicos: diagnosticar la forma en que los supervisores asumen su condición de líder, ya sea de forma positiva o negativa; pronosticar las consecuencias que podrían esperarse a partir de esta forma de asumir el liderazgo, independientemente de la intervención de otras variables, dentro del área de trabajo y actuar a partir del diagnóstico en la dirección adecuada para modificar los aspectos negativos o defectuosos y perfeccionar aquellas en las cuales el liderazgo asume una dirección positiva.

\section{Resultados}

En la Figura 2 se muestra la distribución general de las respuestas de los participantes para analizar el estilo de liderazgo de los directores. En esta figura se observa que el estilo que obtuvo el mayor porcentaje fue el benevolente/ autoritario con un $35 \%$, seguido de autoritario/explorador con un 33\%, consultivo con un $28 \%$ y el participativo con un $4 \%$.

Figura 2. Estilos de liderazgo de los directores

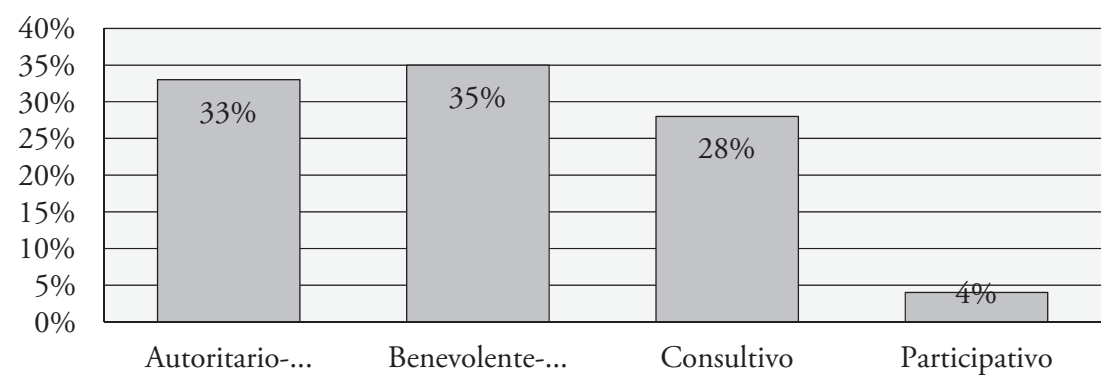

Al presentar los resultados del análisis estadístico para las preguntas, se encontró que los factores que más motivan a los maestros de la generación millennials son: Siento compasión por los necesitados que son incapaces de dar el primer paso para ayudarse (21\%), servir al ciudadano me brinda satisfacciones, aunque no lo agradezcan (19\%) y es difícil para mi el contener mis emociones cuando veo gente en necesidad (18\%). La Tabla 2 detalla estos hallazgos relacionados con los factores que motivan a los maestros de la generación millennials, según lo que contestaron los maestros de la Región Educativa de Ponce, Puerto Rico. 
Tabla 2. Motivación de los empleados de la generación Millennialls con frecuencias y porcentajes

\begin{tabular}{|c|c|c|c|c|c|}
\hline Ítems & $\begin{array}{c}\text { Totalmente } \\
\text { en desacuerdo }\end{array}$ & $\begin{array}{c}\text { Muy en } \\
\text { desacuerdo }\end{array}$ & $\begin{array}{c}\text { De } \\
\text { acuerdo }\end{array}$ & $\begin{array}{l}\text { Muy en } \\
\text { acuerdo }\end{array}$ & $\begin{array}{l}\text { Totalmente } \\
\text { de acuerdo }\end{array}$ \\
\hline $\begin{array}{l}\text { 1. La política es una mala } \\
\text { palabra. }\end{array}$ & $\begin{array}{c}13 \% \\
(22 / 176) \\
\end{array}$ & $\begin{array}{c}49 \% \\
(87 / 176) \\
\end{array}$ & $\begin{array}{c}19 \% \\
(34 / 176)\end{array}$ & $\begin{array}{c}13 \% \\
(23 / 176) \\
\end{array}$ & $\begin{array}{c}6 \% \\
(10 / 176) \\
\end{array}$ \\
\hline $\begin{array}{l}\text { 2. Los vaivenes en la imple- } \\
\text { mentación de la política pú- } \\
\text { blica no me interesan. }\end{array}$ & $\begin{array}{c}18 \% \\
(31 / 176)\end{array}$ & $\begin{array}{c}47 \% \\
(83 / 176)\end{array}$ & $\begin{array}{c}27 \% \\
(48 / 176)\end{array}$ & $\begin{array}{c}5 \% \\
(8 / 176)\end{array}$ & $\begin{array}{c}3 \% \\
(6 / 176)\end{array}$ \\
\hline $\begin{array}{l}\text { 3. Apenas me intereso por los } \\
\text { políticos. }\end{array}$ & $\begin{array}{c}13 \% \\
(23 / 176)\end{array}$ & $\begin{array}{c}38 \% \\
(67 / 176) \\
\end{array}$ & $\begin{array}{c}37 \% \\
(64 / 176)\end{array}$ & $\begin{array}{c}9 \% \\
(16 / 176)\end{array}$ & $\begin{array}{c}3 \% \\
(6 / 176) \\
\end{array}$ \\
\hline $\begin{array}{l}\text { 4. Para mí es difícil interesar- } \\
\text { me en lo que está sucediendo } \\
\text { en mi comunidad. }\end{array}$ & $\begin{array}{c}10 \% \\
(17 / 176)\end{array}$ & $\begin{array}{c}37 \% \\
(66 / 176)\end{array}$ & $\begin{array}{c}42 \% \\
(74 / 176)\end{array}$ & $\begin{array}{c}10 \% \\
(17 / 176)\end{array}$ & $\begin{array}{c}1 \% \\
(2 / 176)\end{array}$ \\
\hline $\begin{array}{l}\text { 5. Contribuyo desinteresada- } \\
\text { mente con mi comunidad. }\end{array}$ & $\begin{array}{c}1 \% \\
(1 / 176) \\
\end{array}$ & $\begin{array}{c}17 \% \\
(30 / 176) \\
\end{array}$ & $\begin{array}{c}58 \% \\
(102 / 176) \\
\end{array}$ & $\begin{array}{c}17 \% \\
(31 / 176) \\
\end{array}$ & $\begin{array}{c}7 \% \\
(12 / 176) \\
\end{array}$ \\
\hline $\begin{array}{l}\text { 6. Un verdadero servicio } \\
\text { público es muy importante } \\
\text { para mí. }\end{array}$ & $\begin{array}{c}3 \% \\
(5 / 176)\end{array}$ & $\begin{array}{c}9 \% \\
(15 / 176)\end{array}$ & $\begin{array}{c}41 \% \\
(34 / 176)\end{array}$ & $\begin{array}{c}30 \% \\
(52 / 176)\end{array}$ & $\begin{array}{c}17 \% \\
(31 / 176)\end{array}$ \\
\hline $\begin{array}{l}\text { 7. Preferiría ver que los fun- } \\
\text { cionarios públicos hacen lo } \\
\text { mejor por mi comunidad, } \\
\text { aunque se vieran afectados } \\
\text { mis propios intereses. }\end{array}$ & $\begin{array}{c}2 \% \\
(3 / 176)\end{array}$ & $\begin{array}{c}25 \% \\
(44 / 176)\end{array}$ & $\begin{array}{c}46 \% \\
(82 / 176)\end{array}$ & $\begin{array}{c}17 \% \\
(30 / 176)\end{array}$ & $\begin{array}{c}10 \% \\
(17 / 176)\end{array}$ \\
\hline $\begin{array}{l}\text { 8. Considero el servicio públi- } \\
\text { co parte de mi deber cívico. }\end{array}$ & $\begin{array}{c}2 \% \\
(4 / 176)\end{array}$ & $\begin{array}{c}17 \% \\
(30 / 176)\end{array}$ & $\begin{array}{c}48 \% \\
(84 / 176) \\
\end{array}$ & $\begin{array}{c}23 \% \\
(41 / 176) \\
\end{array}$ & $\begin{array}{c}10 \% \\
(17 / 176)\end{array}$ \\
\hline $\begin{array}{l}\text { 9. Rara vez me conmuevo } \\
\text { por la súplica de los desam- } \\
\text { parados. }\end{array}$ & $\begin{array}{c}24 \% \\
(43 / 176)\end{array}$ & $\begin{array}{c}42 \% \\
(73 / 176)\end{array}$ & $\begin{array}{c}19 \% \\
(34 / 176)\end{array}$ & $\begin{array}{c}14 \% \\
(24 / 176)\end{array}$ & $\begin{array}{c}1 \% \\
(2 / 176)\end{array}$ \\
\hline $\begin{array}{l}\text { 10. Muchos programas socia- } \\
\text { les son tan importantes para } \\
\text { la sociedad como para dejar- } \\
\text { los desaparecer. }\end{array}$ & $\begin{array}{c}0 \% \\
(0 / 176)\end{array}$ & $\begin{array}{c}7 \% \\
(13 / 176)\end{array}$ & $\begin{array}{c}48 \% \\
(85 / 176)\end{array}$ & $\begin{array}{c}31 \% \\
(54 / 176)\end{array}$ & $\begin{array}{c}14 \% \\
(24 / 176)\end{array}$ \\
\hline $\begin{array}{l}\text { 11. Es difícil para mí el con- } \\
\text { tener mis emociones cuando } \\
\text { veo gente en necesidad. }\end{array}$ & $\begin{array}{c}0 \% \\
(0 / 176)\end{array}$ & $\begin{array}{c}14 \% \\
(24 / 176)\end{array}$ & $\begin{array}{c}41 \% \\
(73 / 176)\end{array}$ & $\begin{array}{c}27 \% \\
(48 / 176)\end{array}$ & $\begin{array}{c}18 \% \\
(31 / 176)\end{array}$ \\
\hline $\begin{array}{l}\text { 12. Para mí, la frase «servir, } \\
\text { honor y patria» estremecen } \\
\text { profundamente mis senti- } \\
\text { mientos. }\end{array}$ & $\begin{array}{c}0 \% \\
(0 / 176)\end{array}$ & $\begin{array}{c}9 \% \\
(16 / 176)\end{array}$ & $\begin{array}{c}48 \% \\
(84 / 176)\end{array}$ & $\begin{array}{c}29 \% \\
(52 / 176)\end{array}$ & $\begin{array}{c}14 \% \\
(24 / 176)\end{array}$ \\
\hline
\end{tabular}




\begin{tabular}{|c|c|c|c|c|c|}
\hline Ítems & $\begin{array}{c}\text { Totalmente } \\
\text { en desacuerdo }\end{array}$ & $\begin{array}{c}\text { Muy en } \\
\text { desacuerdo }\end{array}$ & $\begin{array}{c}\text { De } \\
\text { acuerdo }\end{array}$ & $\begin{array}{l}\text { Muy en } \\
\text { acuerdo }\end{array}$ & $\begin{array}{l}\text { Totalmente } \\
\text { de acuerdo }\end{array}$ \\
\hline $\begin{array}{l}\text { 13. Pienso en el bienestar de } \\
\text { las personas que no conozco. }\end{array}$ & $\begin{array}{c}2 \% \\
(4 / 176)\end{array}$ & $\begin{array}{c}11 \% \\
(20 / 176)\end{array}$ & $\begin{array}{c}52 \% \\
(91 / 176)\end{array}$ & $\begin{array}{c}26 \% \\
(46 / 176)\end{array}$ & $\begin{array}{c}9 \% \\
(15 / 176)\end{array}$ \\
\hline $\begin{array}{l}\text { 14. Las cosas que pasan dia- } \\
\text { riamente me hacen recordar } \\
\text { lo dependientes que somos } \\
\text { unos de otros. }\end{array}$ & $\begin{array}{c}1 \% \\
(2 / 176)\end{array}$ & $\begin{array}{c}14 \% \\
(24 / 176)\end{array}$ & $\begin{array}{c}40 \% \\
(71 / 176)\end{array}$ & $\begin{array}{c}30 \% \\
(52 / 176)\end{array}$ & $\begin{array}{c}15 \% \\
(27 / 176)\end{array}$ \\
\hline $\begin{array}{l}\text { 15. Siento compasión por los } \\
\text { necesitados que son incapaces } \\
\text { de dar el primer paso para } \\
\text { ayudarse. }\end{array}$ & $\begin{array}{c}1 \% \\
(2 / 176)\end{array}$ & $\begin{array}{c}13 \% \\
(23 / 176)\end{array}$ & $\begin{array}{c}32 \% \\
(57 / 176)\end{array}$ & $\begin{array}{c}32 \% \\
(57 / 176)\end{array}$ & $\begin{array}{c}21 \% \\
(37 / 176)\end{array}$ \\
\hline $\begin{array}{l}\text { 16. Hay algunos programas y } \\
\text { políticas públicas que ayuda- } \\
\text { ría de corazón. }\end{array}$ & $\begin{array}{c}1 \% \\
(2 / 176)\end{array}$ & $\begin{array}{c}7 \% \\
(12 / 176)\end{array}$ & $\begin{array}{c}52 \% \\
(93 / 176)\end{array}$ & $\begin{array}{c}27 \% \\
(47 / 176)\end{array}$ & $\begin{array}{c}13 \% \\
(22 / 176)\end{array}$ \\
\hline $\begin{array}{l}\text { 17. Hacer la diferencia en la } \\
\text { sociedad tiene más significado } \\
\text { para mí que los logros perso- } \\
\text { nales que pueda alcanzar. }\end{array}$ & $\begin{array}{c}3 \% \\
(5 / 176)\end{array}$ & $\begin{array}{c}16 \% \\
(28 / 176)\end{array}$ & $\begin{array}{c}48 \% \\
(85 / 176)\end{array}$ & $\begin{array}{c}22 \% \\
(39 / 176)\end{array}$ & $\begin{array}{c}11 \% \\
(19 / 176)\end{array}$ \\
\hline $\begin{array}{l}\text { 18. Creo en anteponer el } \\
\text { servicio a mis necesidades. }\end{array}$ & $\begin{array}{c}6 \% \\
(11 / 176)\end{array}$ & $\begin{array}{c}33 \% \\
(58 / 176)\end{array}$ & $\begin{array}{c}39 \% \\
(68 / 176)\end{array}$ & $\begin{array}{c}17 \% \\
(30 / 176)\end{array}$ & $\begin{array}{c}5 \% \\
(9 / 176)\end{array}$ \\
\hline $\begin{array}{l}\text { 19. Mejorar económicamente } \\
\text { es más importante para mí } \\
\text { que hacer una buena obra. }\end{array}$ & $\begin{array}{c}6 \% \\
(10 / 176)\end{array}$ & $\begin{array}{c}29 \% \\
(51 / 176)\end{array}$ & $\begin{array}{c}33 \% \\
(59 / 176)\end{array}$ & $\begin{array}{c}17 \% \\
(30 / 176)\end{array}$ & $\begin{array}{c}15 \% \\
(26 / 176)\end{array}$ \\
\hline $\begin{array}{l}\text { 20. Mucho de lo que hago es } \\
\text { por una causa más importante } \\
\text { que yo mismo/a. }\end{array}$ & $\begin{array}{c}2 \% \\
(4 / 176)\end{array}$ & $\begin{array}{c}23 \% \\
(41 / 176)\end{array}$ & $\begin{array}{c}47 \% \\
(82 / 176)\end{array}$ & $\begin{array}{c}23 \% \\
(41 / 176)\end{array}$ & $\begin{array}{c}5 \% \\
(8 / 176)\end{array}$ \\
\hline $\begin{array}{l}\text { 21. Siento que las personas } \\
\text { deben aportar a la sociedad } \\
\text { más de lo que reciben de ella. }\end{array}$ & $\begin{array}{c}2 \% \\
(3 / 176)\end{array}$ & $\begin{array}{c}33 \% \\
(58 / 176)\end{array}$ & $\begin{array}{c}35 \% \\
(62 / 176)\end{array}$ & $\begin{array}{c}23 \% \\
(41 / 176)\end{array}$ & $\begin{array}{c}7 \% \\
(12 / 176)\end{array}$ \\
\hline $\begin{array}{l}\text { 22. Servir al ciudadano me } \\
\text { brinda satisfacciones, aunque } \\
\text { no lo agradezcan. }\end{array}$ & $\begin{array}{c}1 \% \\
(2 / 176)\end{array}$ & $\begin{array}{c}9 \% \\
(15 / 176)\end{array}$ & $\begin{array}{c}44 \% \\
(78 / 176)\end{array}$ & $\begin{array}{c}27 \% \\
(47 / 176)\end{array}$ & $\begin{array}{c}19 \% \\
(34 / 176)\end{array}$ \\
\hline $\begin{array}{l}\text { 23. Soy una de esas personas } \\
\text { que se pondrían en riesgo } \\
\text { para ayudar a otras personas. }\end{array}$ & $\begin{array}{c}5 \% \\
(9 / 176)\end{array}$ & $\begin{array}{c}24 \% \\
(42 / 176)\end{array}$ & $\begin{array}{c}43 \% \\
(75 / 176)\end{array}$ & $\begin{array}{c}21 \% \\
(37 / 176)\end{array}$ & $\begin{array}{c}7 \% \\
(13 / 176)\end{array}$ \\
\hline $\begin{array}{l}24 . \text { Estoy preparada/o para } \\
\text { realizar grandes sacrificios por } \\
\text { el bienestar de la sociedad. }\end{array}$ & $\begin{array}{c}6 \% \\
(11 / 176)\end{array}$ & $\begin{array}{c}28 \% \\
(48 / 176)\end{array}$ & $\begin{array}{c}39 \% \\
(69 / 176)\end{array}$ & $\begin{array}{c}18 \% \\
(32 / 176)\end{array}$ & $\begin{array}{c}9 \% \\
(16 / 176)\end{array}$ \\
\hline
\end{tabular}

Fuente: Elaboración propia a partir de Statistical Package for the Social Sciences. 
Para discutir la cuarta pregunta: ¿Cuál es la relación entre el estilo de liderazgo del director con la motivación de los empleados de la generación millennials?, se utilizó la estadística correlacional de Rho de Spearman. Esta prueba se seleccionó para someter a prueba la hipótesis nula: no existe relación significativa entre el estilo de liderazgo del director con la motivación de los empleados de la generación millennials, según los docentes de la Región Educativa de Ponce. Para propósitos de análisis, los estilos se codificaron de la siguiente forma: 4 (benevolente/autoritario), 3 (autoritario/explorador), 2 (consultivo) y 1 (participativo).

De acuerdo con los resultados de la prueba estadística, Rho de Spearman = $-361, p=.000$. Este valor de $p$, si se compara a un nivel de significancia de 0.05 $(p<0.05)$, establece que se rechaza la hipótesis nula. Por tanto, existe relación significativa entre el estilo de liderazgo del director con la motivación de los empleados de la generación millennials, según los docentes y las docentes de la Región Educativa de Ponce. De acuerdo con la tabla de valores, se considera que la relación entre ambas variables es significativa y se puede considerar moderada de acuerdo con la clasificación de Field (2018). La Tabla 3 muestra los resultados de la prueba estadística Rho de Spearman.

Tabla 3. Prueba de correlación de Rho de Spearman

\begin{tabular}{lllcc}
\hline & & $\begin{array}{c}\text { Estilo de } \\
\text { liderazgo }\end{array}$ & Motivación \\
\hline $\begin{array}{l}\text { Rho de } \\
\text { Spearman }\end{array}$ & \multirow{2}{*}{ Estilo de liderazgo } & Coeficiente de correlación & 1.000 & $-.361^{* *}$ \\
& & Sig. (bilateral) & --- & .000 \\
& & $\mathrm{~N}$ & 176 & 176 \\
\hline \multirow{3}{*}{ Motivación } & Coeficiente de correlación & $-.361^{* *}$ & 1.000 \\
& & Sig. (bilateral) & .000 &.--- \\
& & $\mathrm{N}$ & 176 & 176 \\
\hline
\end{tabular}

Nota: **La correlación es significativa en el nivel 0.01 ( 2 colas).

Fuente: Elaboración propia a partir de Statistical Package for the Social Sciences.

La prueba de correlación de Rho de Spearman provee un análisis entre variables ordinales (Field, 2018). Esta prueba demuestra que la dirección de la relación entre el estilo de liderazgo del director con la motivación de los empleados de la generación millennials es negativa con un coeficiente de -0.361. Ello significa que, a medida que los maestros tienen directores con estilos benevolente/autoritario y autoritario/explorador, la motivación disminuye significativamente. 


\section{Discusión}

En esta investigación se evidenció que los directores con estilo autoritario obtuvieron un mayor porcentaje que los estilos consultivos y participativos. El liderazgo autoritario aporta elementos que son necesarios en la gerencia educativa como la capacidad para expresar las ideas de forma directa; el tomar decisiones sin dudar hacerlo cuando es necesario, por incómodas y antipáticas que resulten las mismas; la gran capacidad de respuesta ante las necesidades de la institución educativa, al mismo tiempo, que la eficacia en esa capacidad; el poder ofrecer soluciones rápidas; la firmeza del gerente al tomar decisiones y la constante y firme supervisión de los trabajadores.

Sin embargo, debe mencionarse que el líder autoritario concentra todo el poder en sí mismo y es él quien toma las decisiones. Dado que en esta investigación se encontró que el estilo predominante de los directores es el autoritario, se entiende que los maestros y maestras no toman decisiones dentro de la institución educativa a la cual pertenecen, sino que solo obedecen y hacen lo que el líder les dice. Por tanto, que este hallazgo discrepa de las estrategias que prefieren los maestros y maestras millennials, dado que en la pregunta anterior se discutió que los maestros se motivan cuando se involucran en los procesos administrativos, cuando se les da confianza, son escuchados y cuando se incorporan sus ideas en distintas situaciones.

El hallar que los directores participantes tengan un estilo benevolentelautoritario con un 35\%, seguido de autoritario/explorador con un 33\%, confirma que existen características que pueden ser favorables para las instituciones educativas. Estos resultados discrepan de las características que debe tener un líder. Una de ellas se relaciona con la Teoría Transformacional de Liderazgo de Bass (1985) sobre el liderazgo transformacional, según la cual el estilo de liderazgo transformacional proporciona nuevas ideas y estimula intelectualmente a sus empleados ayudando a que se produzcan cambios positivos en las organizaciones e instituciones, puesto que el liderazgo transformacional reconoce y potencia a los miembros de la organización.

Lewin, Lippitt y White (1939) realizaron estudios experimentales en los que se establecieron diferencias entre el estilo de liderazgo autoritario versus el democrático. El autoritario dicta lo que se tiene que hacer y no se preocupa por el desarrollo del grupo, mientras que el democrático (permisivo) toma en cuenta al grupo y sus necesidades. Según estos autores, el estilo de vida y pensamiento motivado por el líder domina las relaciones entre el grupo y con el líder. Asimismo, en la práctica del liderazgo autocrático, era 
común la actitud hostil y marcadamente personal en lugar de una actitud cooperativa.

La investigación de Pozzi (2013) también exploró los factores que buscan los millennials en sus trabajos. Los resultados acerca de las expectativas laborales de los millennials demostraron una tendencia a elegir trabajos que sean interesantes y desafiantes. Esto es interesante discutirlo, dado que la profesión de maestro o docente reúne estas características. En la investigación de Pozzi (2013), también se menciona que los millennials le adjudican importancia a las oportunidades de crecimiento que ofrecen las empresas en las que eligen trabajar o en la que estén trabajando. Este aspecto llama la atención, puesto que, en los resultados de la investigación se muestra total importancia en servir al ciudadano para tener satisfacciones. Sin embargo, en la investigación de Pozzi (2013), se hace alusión a que la empresa les ofrezca un buen nivel salarial con beneficios adicionales a los millennials.

Otro autor que también auscultó la motivación laboral de los docentes universitarios en la Universidad Rafael Belloso Chacín, en Venezuela, fue Añez (2006). Evidenció la existencia de pocos incentivos que cubran las expectativas y estima de los docentes, no existiendo una correspondencia directa entre los logros y el reconocimiento; es decir, a mayores logros alcanzados, deberían corresponder mayores reconocimientos, lo cual está desmotivando a los docentes.

Estos hallazgos no reflejan el sentir de los maestros y maestras encuestados en esta investigación. En consonancia con el hallazgo de esta investigación se encuentra la de Ruiz (2011), quien estudió la influencia del estilo de liderazgo del director en la eficacia de las instituciones educativas. Los resultados afirmaron que el estilo de liderazgo del director influye significativamente en la eficacia de las instituciones educativas del Consorcio. Otra investigación donde se encontró una relación entre el estilo del director y el desempeño pedagógico fue la de Raxuleu (2014), quien demostró que existe un alto vínculo entre el liderazgo del director y el desempeño pedagógico docente. Estas dos investigaciones mencionadas presentan cómo el estilo de liderazgo se relaciona con variables en los maestros. Sin embargo, hay que discutir que, en esta investigación, la relación fue negativa hacia el estilo autoritario.

Miskel, Fewer y Stewart (2009) expresaron que es importante establecer cómo el trabajo de todos los maestros y la forma en que los directores ejercen su liderazgo guarda relación para crear las condiciones que propicien un clima organizacional satisfactorio. El estilo participativo en esta investigación fue el menos encontrado en los directores de la región educativa 
de Ponce, con un solo un 4\%. Nir y Kranot (2006) hallaron una relación entre el estilo de liderazgo y la eficiencia personal del maestro. Estos seleccionaron el modelo de liderazgo transformativo y la eficiencia personal de los maestros. El estudio concluyó que el estilo de liderazgo no tuvo relación con la eficiencia general del profesorado, pero reflejó las características organizacionales. También, indicaron que la autoeficacia de los docentes es uno de los factores más influyentes en la calidad de la enseñanza y la motivación de los docentes.

\section{Conclusiones}

Los resultados de esta investigación demuestran la importancia de determinar cómo se relaciona el estilo de liderazgo del director escolar con la motivación laboral de los empleados de la generación millennials. La investigación ha demostrado que existen muchos factores motivacionales en los maestros y maestras que necesitan ser atendidos en el Departamento de Educación de Puerto Rico; además, que el estilo de liderazgo predominante de los directores es el autoritario. El estudio ha permitido proveer información sobre una base científica dentro del sistema educativo puertorriqueńo, específicamente relacionada con los estilos de liderazgo y los factores motivacionales de los maestros y maestras; por lo que esta investigación contiene información que sirve para la toma de decisiones relacionadas con la implantación de prácticas de liderazgo. Los hallazgos obtenidos llevaron a concluir lo siguiente:

1. Los estilos de liderazgo de los directores de las escuelas de una Región Educativa en Puerto Rico más predominantes son el benevolente/autoritario con un $35 \%$, seguido de autoritario/explorador con un 33\%.

2. Los factores que más motivan a los maestros de la generación millennials son: sentir compasión por los necesitados que son incapaces de dar el primer paso para ayudarse (21\%), servir al ciudadano me brinda satisfacciones, aunque no lo agradezcan (19\%) y contener mis emociones cuando veo gente en necesidad (18\%).

3. Existe relación significativa entre el estilo de liderazgo del director con la motivación de los empleados de la generación millennials, según los docentes de la Región Educativa de Ponce. Esto representa que a medida que los maestros tienen directores con estilos benevolente/autoritario y autoritario/explorador, la motivación disminuye significativamente. 


\section{REFERENCIAS BIBLIOGRÁFICAS}

Agüero, S. (2015). ¿De qué manera desafían los milleniales el liderazgo tradicional? Recuperado de https://revistaitnow.com/manera-los-millennialsdesafian-liderazgo-tradicional

Añez, S. (2006). Cultura organizacional y motivación laboral de los docentes universitarios. Maracaibo, Venezuela: Universidad Rafael Belloso Chacín.

Archer, D. y Cameron, A. (2008). Liderazgo cooperativo - cómo tener éxito en el interconectado mundo. Butterworth Heinemann.

Archer, D. y Cameron, A. (2013). Collaborative Leadership. Building relationships, handling conflicts and sharing control ( $2^{\text {nd }}$ ed.). Londres: Routledge. https:// doi.org/10.4324/9780203067505

Avolio, B., Bass, B. y Jung, D. (1996). Replicated confirmatory factor analysis of the Multifactor Leader Questionnaire (Form 5X). Binghamton: State University of New York at Binghamton, Center for Leadership Studies.

Avolio, B. y Yammarino, F. (1990). Operationalizing charismatic leadership using a levels-of-analysis framework. Leadership Quarterly, 1(3), 193-208. https://doi.org/10.1016/1048-9843(90)90020-I

Badford, L. y Lippitt, R. (2005). Building a democratic work group. Management and Philosophy, 22(3), 142-173.

Bass, B. (1985). Leadership and Performance Beyond Expectations. Nueva York: Division of Macmillan, Inc.

Bass, B. y Avolio, B. (1994). Improving organizational effectiveness through transformational leadership. Thousand Oaks, CA: Sage Publications.

Broido, E. (2004). Understanding Diversity in Millennials Students. New Directions for Students Services, (106), 73-85. https://doi.org/10.1002/ss.126

Creswell, J. W. y Creswell, J. D. (2017). Research Design Qualitative, Quantitative, and Mixed Methods Approaches ( $5^{\text {th }}$ edition). Sage publications.

Del Valle, I. (2010). Liderazgo distributivo: una nueva visión del liderazgo educativo. Recuperado de https://www.researchgate.net/publication/282133831

Field, A. (2018). Discovering Statistics using IBM SPSS Statistics ( $5^{\text {th }}$ edition). Sage Publications, Estados Unidos.

Hernández Sampieri, R y Mendoza, C. (2018). Metodología de la investigación: las rutas cuantitativa, cualitativa y mixta. México, DF: McGraw Hill Interamericana Ediciones.

Hersey, P. y Blanchard, K. H. (1977). So you want to know your leadership style? Training and Development Journal, 28(2), 22-37. 
Herzberg, Frederick. (1968). One More Time: How do you Motivate Employees. Recuperado de https://kyleshulfermba530.weebly.com/uploads/2/3/4/5/ 23454770/one_more_time_-_how_do_you_motivate_employees.pdf

Krejcie, R. V. y Morgan, D. W. (1970). Determining sample size for research activities. Educational and Psychological Measurement, 30(3), 607-610. https:// doi.org/10.1177/001316447003000308

Lewin, K., Lippitt, R. y White, R. K. (1939). Patterns of aggressive behavior in experimentally created «social climates». The Journal of Social Psychology, 10(2), 269-299. https://doi.org/10.1080/00224545.1939.9713366

Lowe, D., Levitt, K. y Wilson, T. (2008). Solutions for Retaining Generation Y Employees in the Workplace. Business Renaissance Quarterly, 43.

Lupano, M. y Castro, A. (2005). Estudios sobre el liderazgo: teoría y evaluación. Psicodebate, 6, 107-122. https://doi.org/10.18682/pd.v6i0.444

Lussier, R. y Achua, C. (2016). Liderazgo: teoría, aplicación, desarrollo de habilidades (sexta edición). México: Thomson.

Miskel, A, Fewer, D. y Stewart, O. (2009). El liderato administrativo en la toma de decisiones. España, Universidad de Alcalá.

Naile, I. y Selesho, J. (2014). The Role of Leadership in Employee Motivation. Mediterranean Journal of Social Sciences, 5(3), 175-182. https://doi. org/10.5901/mjss.2014.v5n3p175

Naim, M. F. y Lenka, U. (2018). Development and retention of Generation Y employees: a conceptual framework. Employee Relations, 40(2), 433-455. https://doi.org/10.1108/ER-09-2016-0172

Nir, A. y Kranot, N. (2006). School principal's leadership style and teachers' selfefficacy. Planning and Changing, 37(3-4), 205-218.

Ortega, Gasset J. (1983). El tema de nuestro tiempo. Madrid: Alianza Editorial / Revista de Occidente.

Pozzi, S. (2013). Generación "Y»: Sus expectativas laborales (Tesis de Grado). Universidad de San Andrés, Buenos Aires.

Ramírez, R., Abreu, J. y Badii, M. (2008). La motivación laboral, factor fundamental para el logro de objetivos organizacionales: Caso empresa manufacturera de tubería de acero. Daena: International Journal of Good Conscience, 3(1), 143-185.

Raxuleu, M. (2014). Liderazgo del director y desempeño pedagógico docente (Tesis de Grado). Universidad Rafael Landívar, Quetzaltenango, Guatemala.

Rubin, H. (2009). Liderazgo colaborativo: Desarrollo de asociaciones eficaces para las comunidades y escuelas. Thousand Oaks, CA: Corwin Press. 
Ruiz, G. (2011). Influencia del estilo de liderazgo del director en la eficacia de las instituciones educativas del consorcio de Santo Domingo de Guzmán de Lima Norte. (Tesis de Posgrado). Lima: Universidad Nacional Mayor de San Marcos.

Strauss, W. y Howe, N. (1991). Generations: The history of America's future, 1584 to 2069. New York: William Morrow.

Stanford, E. (2017). 5 cosas que CREES saber de los Millennials que son probablemente erróneas. Recuperado de http://www.queretaro-dale-carnegie.com/ blog/5-cosas-que-crees-saber-de-los-millennials-pero-son-probablemente-erroneas

Torres, J. (2010). La justicia curricular. El caballo de Troya de la cultura escolar. Madrid: Morata.

Vega, C. y Zavala, G. (2004). Adaptación del cuestionario multifactorial del liderazgo (MLQ Forma $5 X$ corta de B. Bass y B. Avolio al contexto organizacional chileno. Recuperado de http://repositorio.uchile.cl/tesis/uchile/2004/ vega_c/sources/vega_c.pdf

Williamson, R. y Meyer-Looze, C. (2010). Working with Gen Y Teachers: Dealing with a Changing Teacher Workforce. Pittsburgh: Education Partnerships, Inc. 